Adelaide Univ. Preprint: ADP-93-211/T129

\title{
Charge-Symmetry Breaking, Rho-Omega Mixing, and the Quark Propagator
}

\author{
G. Krein ${ }^{a}$, A.W. Thomas ${ }^{b}$ and A.G. Williams ${ }^{b, c}$ \\ ${ }^{a}$ Instituto de Física Téorica, UNESP \\ Rua Pamplona 145, 01405 São Paulo, SP, Brazil \\ ${ }^{b}$ Department of Physics and Mathematical Physics, University of Adelaide \\ GPO Box 498 Adelaide, S. Aust. 5001, Australia \\ ${ }^{c}$ Department of Physics and the Supercomputer Computations Research Institute \\ Florida State University, Tallahassee,FL 32306, USA
}

\begin{abstract}
The momentum-dependence of the $\rho^{0}-\omega$ mixing contribution to chargesymmetry breaking (CSB) in the nucleon-nucleon interaction is compared in a variety of models. We focus in particular on the role that the structure of the quark propagator plays in the predicted behaviour of the $\rho^{0}-\omega$ mixing amplitude. We present new results for a confining (entire) quark propagator and for typical propagators arising from explicit numerical solutions of quark Dyson-Schwinger equations. We compare these to hadronic and free quark calculations. The implications for our current understanding of CSB experiments is discussed.
\end{abstract}

Manuscript available as a $\mathrm{HT}_{\mathrm{E}} \mathrm{X}$ file

e-mail addresses:

gkrein@ift.uesp.ansp.br

athomas@physics.adelaide.edu.au 
awilliam@physics.adelaide.edu.au 
A wide variety of charge symmetry breaking (CSB) phenomena in nuclear physics appear to be well explained in terms of one-boson exchange potentials, when electromagnetic effects, the neutron-proton mass difference, and isoscalar-isovector meson mixing are included. A recent and extensive review of CSB has recently appeared [1]. Ultimately we would like to understand CSB in terms of electromagnetic effects and the $u-d$ quark mass difference in a microscopic quantum chromodynamics (QCD) based description of the strong interactions. Any potential which gives rise to CSB in the nucleon-nucleon $(N N)$ sytem can be categorized as either a class III or class IV interaction in the conventions of Henley and Miller [2].

In the relatively successful explanation of a number of observed CSB phenomena, calculated contributions from $\rho^{0}-\omega$ mixing play a major role. These phenomena include: (i) differences between $p p$ and $n n$ scattering lengths and effective range parameters, (ii) binding energy difference of ${ }^{3} \mathrm{H}$ and ${ }^{3} \mathrm{He}$, (iii) analyzing power difference in $n$ - $p$ elastic scattering, and (iv) the Nolen-Schiffer anomaly. In the one-boson exchange model of the nucleon-nucleon interaction the $\rho^{0}-\omega$ potential is generated by $\rho^{0}-\omega$ mixing in the intermediate vectormeson propagators [3]. Another source of CSB arising from meson mixing is that from $\pi^{0}-\eta$ mixing, but this is typically much less important than that due to the $\rho^{0}$ and $\omega$. The long standing problem of explaining the binding energy differences of mirror nuclei, (the Nolen-Schiffer anomaly) [4, appears to have been largely resolved as a result of the $\rho^{0}-\omega$ mixing potential[5]. The theoretical understanding of the difficult neutron-proton analyzing power difference measurements carried out at TRIUMF [6] and IUCF[7] relied on $\rho^{0}-\omega$ mixing and in particular this was the dominant contribution in the latter. [8, 9, 10].

Although the situation appears to be very satisfactory, there is however at least one potential problem with the standard evaluations which has been pointed out recently by Goldman, Henderson and Thomas(GHT)[11. The 
problem is associated with the assumed off-shell behavior of the $\rho^{0}-\omega$ mixing matrix element. The standard assumption is that the mixing amplitude is momentum independent with its value extracted from the experimental data on $e^{+} e^{-} \rightarrow \pi^{+} \pi^{-}$at the $\omega$ pole, $q^{2} \simeq m_{\omega}^{2}$, while the exchanged mesons in Eq. (5) have space-like four-momentum, $q^{2}<0$, and are therefore highly virtual. In their initial study a simple model was used where the vector mesons are considered as quark-antiquark composites. Because of the the up-down quark mass difference the $\rho^{0}-\omega$ mixing amplitude is generated by an intermediate quark loop. A $\rho^{0}$ of four momentum $q^{\mu}$ dissociates into a quark-antiquark pair and then recombines to form an $\omega$, or vice versa. The quark and antiquark momenta are $\ell^{\mu}+\left(q^{\mu} / 2\right)$ and $-\ell^{\mu}+\left(q^{\mu} / 2\right)$ respectively, where $\ell$ is the loop momentum. There will also be a contribution from electromagnetic effects, but this will be much smaller than that from the quark mass difference. Simple vertex functions were introduced and free quark propagators were used. Once the mixing amplitude was calculated, GHT obtained the coordinate space (static) potential by Fourier transforming the resulting momentum-space potential. The basic conclusions of the GHT calculation were: (i) the mixing amplitude is strongly momentum dependent, (ii) there is a node in the potential at about $0.9 \mathrm{fm}$ and, (iii) because of the node, the potential changes sign and so its importance is greatly suppressed.

The potential implications of this result of GHT are profound in view of the central role meson mixing plays in our understanding of charge symmetry breaking. If these results can be confirmed in other more realistic calculations, it may be necessary to find new sources of CSB in order to explain existing data. Since the initial investigation by GHT other studies appear to support their basic conclusions. These include investigations of $\pi^{0}-\eta$ mixing using a quark loop model[12], chiral perturbation theory[13], and a hadronic model[14]. In addition, each of two further studies of $\rho^{0}-\omega$ mixing using a hadronic model 15 
and using QCD sum rules 16 also support the basic conclusions of GHT.

In the present study we investigate the role of quark confinement and the nature of the quark propagator in a $q-\bar{q}$ based description of the $\rho^{0}-\omega$ mixing amplitude. This study is motivated by one of the difficulties associated with the GHT calculation, namely that, as their work did not include quark confinement, an unphysical $q \bar{q}$-pair production threshold resulted in the timelike region - at $q^{2}=4 m_{q}^{2}$. This corresponds to the vector meson being able to dissociate into an on-shell quark-antiquark pair for $q^{2} \geq 4 m_{q}^{2}$. We review some necessary formalism before discussing details of our quark propagators.

The interaction Lagrangian densities for the nucleon- $\rho$ and nucleon- $\omega$ couplings are

$$
\begin{gathered}
\mathcal{L}_{N N \rho}=g_{\rho} \bar{\psi} \gamma^{\mu} \vec{\tau} \cdot \psi \vec{\rho}_{\mu}+f_{\rho} \bar{\psi} \sigma^{\mu \nu} \vec{\tau} \cdot \psi \frac{\partial_{\mu}}{2 M} \vec{\rho}_{\nu}, \\
\mathcal{L}_{N N \omega}=g_{\omega} \bar{\psi} \gamma^{\mu} \psi \omega_{\mu} .
\end{gathered}
$$

These definitions are standard, (although some authors vary in their definitions of the couplings by including additional factors of $1 / 2$ ). No tensor coupling for the $N N \omega$ vertex is included since we choose typical coupling constants and form factors determined by the Bonn group [17]. The contribution from $\rho^{0}-\omega$ mixing to the $N N$ potential, is given by (with $\Gamma^{\mu} \equiv i \sigma^{\mu \nu} q_{\nu} / 2 M_{N}$ and $\left.C_{\rho} \equiv f_{\rho} / g_{\rho}\right)$

$$
\begin{aligned}
& \hat{V}_{I I I}^{\rho \omega}(q)=V(q) \gamma^{\mu}(1) \gamma_{\mu}(2)\left[\tau_{z}(1)+\tau_{z}(2)\right] \\
& \hat{V}_{I V}^{\rho \omega}(q)=V(q) C_{\rho}\left[\Gamma^{\mu}(1) \gamma_{\mu}(2) \tau_{z}(1)-\gamma^{\mu}(1) \Gamma_{\mu}(2) \tau_{z}(2)\right]
\end{aligned}
$$

where

$$
V(q)=-\frac{g_{\rho} g_{\omega} F_{N \rho}\left(q^{2}\right) F_{N \omega}\left(q^{2}\right)\left\langle\rho^{0}|H| \omega\right\rangle}{\left(q^{2}-m_{\rho}^{2}\right)\left(q^{2}-m_{\omega}^{2}\right)},
$$

where $\left\langle\omega|H| \rho^{0}\right\rangle$ is the meson mixing amplitude as defined in Ref. [3]. We have introduced the standard Bonn form factors at the nucleon-meson vertices, which for on-shell nucleons are given by $F_{N \rho}\left(q^{2}\right) \equiv 1 /\left[1-\left(q^{2} / \Lambda_{\rho}^{2}\right)\right]$ and $F_{\omega}\left(q^{2}\right) \equiv$ 
$1 /\left[1-\left(q^{2} / \Lambda_{\omega}^{2}\right)\right]$. With this normalization for the form factors, the appropriate Bonn couplings are $g_{\rho}^{2} / 4 \pi=0.41$ and $g_{\omega}^{2} / 4 \pi=10.6$. Also, $\Lambda_{\rho}=1400 \mathrm{MeV}$, $\Lambda_{\omega}=1500 \mathrm{MeV}, C_{\rho} \equiv f_{\rho} / g_{\rho}=6.1$ and as already stated $C_{\omega} \equiv f_{\omega} / g_{\omega}=0$.

Our starting point for the present calculations is the expression for the $\rho \omega$ mixing self-energy in terms of a quark-antiquark loop insertion

$\Pi^{\mu \nu}\left(q^{2}\right)=3 i g_{q \rho} g_{q \omega} \int \frac{d^{4} \ell}{(2 \pi)^{4}} \operatorname{tr}\left[\Gamma_{\rho}^{\mu}\left(\ell_{+}, \ell_{-}\right) S_{u}\left(l_{-}\right) \Gamma_{\omega}^{\nu}\left(\ell_{-}, \ell_{+}\right) S_{u}\left(\ell_{+}\right)\right]-[u \longrightarrow d]$.

The trace is over spinor indices and the factor of three comes having performed the colour trace. $\Gamma_{\rho}^{\mu}\left(p^{\prime}, p\right)$ and $\Gamma_{\omega}^{\mu}\left(p^{\prime}, p\right)$ are the quark-vector-meson vertex functions for the $\rho^{0}$ and $\omega$ respectively, $S_{i}(q)$ is the quark propagator with flavour $i(i=u, d)$ and $\ell_{ \pm} \equiv \ell \pm(q / 2)$. We take for simplicity here $\Gamma_{\rho}^{\mu}\left(p^{\prime}, p\right)=\gamma^{\mu} F_{q \rho}\left(p^{\prime}, p\right)$ and $\Gamma_{\omega}^{\mu}\left(p^{\prime}, p\right)=\gamma^{\mu} F_{q \omega}\left(p^{\prime}, p\right)$, where $F_{q \rho}$ and $F_{q \omega}$ are the quark-vector-meson form factors. Since we wish to study the $q^{2}$-dependence arising from the quark propagator in the loop, we follow Ref. [11] and consider these form factors to be functions of $\ell^{2}$ only, i.e., $F_{q \rho}\left(\ell_{+}, \ell_{-}\right)=F_{q \rho}\left(\ell^{2}\right)$ and $F_{q \omega}\left(\ell_{-}, \ell_{+}\right)=F_{q \omega}\left(\ell^{2}\right)$. We do not wish to introduce any unconstrained $q^{2}$-dependent behaviour through the quark-meson vertex functions.

We can make use of Lorentz invariance and current conservation (i.e., $\left.q_{\mu} \Pi^{\mu \nu}=0\right)$ to write $\Pi^{\mu \nu} \equiv\left[-g^{\mu \nu}+\left(q^{\mu} q^{\nu} / q^{2}\right)\right] \Pi\left(q^{2}\right)$, where this is the definition of the scalar function $\Pi\left(q^{2}\right)$. Note that in the case of the photon self-energy we would require $\Pi(0)=0$ in order that the photon not acquire a mass through self-energy insertions, however there is no such restriction in the present case. Since we are only interested in coupling to conserved external currents, (i.e., since $q_{\mu} J^{\mu}=0$ for the nucleons), we need only retain the $g^{\mu \nu} \Pi\left(q^{2}\right)$ part of Eq. (6) in our calculation of the $N N$-interaction. A comparison of the definition [a] of the mixing amplitude $\left\langle\omega|H| \rho^{0}\right\rangle$ with the usual Feyman rules shows that this is identical with the $\rho \omega$ mixing self-energy $\Pi\left(q^{2}\right)$, i.e., $\left\langle\omega|H| \rho^{0}\right\rangle \equiv \Pi\left(q^{2}\right)$. 
The general form of the quark propagator in Minkowski space is

$$
S(q) \equiv \frac{1}{A\left(q^{2}\right) \not q-B\left(q^{2}\right)} \equiv \frac{Z\left(q^{2}\right)\left[\not q+M\left(q^{2}\right)\right]}{q^{2}-M^{2}\left(q^{2}\right)} \equiv F\left(q^{2}\right)\left[\not q+M\left(q^{2}\right)\right],
$$

where $A\left(q^{2}\right)$ and $B\left(q^{2}\right)$ are scalar functions of $q^{2}$ and, $Z\left(q^{2}\right)=1 / A\left(q^{2}\right)$ and $M\left(q^{2}\right)=B\left(q^{2}\right) / A\left(q^{2}\right)$. One possible mechanism of quark confinement is that the quark propagator does not have a mass pole, i.e., that the function $F\left(q^{2}\right) \equiv Z\left(q^{2}\right) /\left[q^{2}-M^{2}\left(q^{2}\right)\right]$ does not have a pole or is an entire function in the complex $q^{2}$ plane[18]. One sees that there is no mass pole when the function $Z\left(q^{2}\right)$ goes to zero for $q^{2} \longrightarrow M^{2}\left(q^{2}\right)$. One explicit quark model where this occurs can be found in Ref ([19]) where this property arises from the solution of a model quark Dyson-Schwinger equation.

We can isolate the $g^{\mu \nu} \Pi\left(q^{2}\right)$ part of $\Pi^{\mu \nu}$ and we then find in our $q \bar{q}$-model that the mixing amplitude can be written as

$$
\begin{aligned}
& \Pi\left(q^{2}\right)=3 i g_{q \rho} g_{q \omega} \int \frac{d^{4} \ell}{(2 \pi)^{4}} F_{q \rho}\left(\ell^{2}\right) F_{q \omega}\left(\ell^{2}\right)\left\{\left[F_{u}\left(\ell_{-}^{2}\right) F_{u}\left(\ell_{+}^{2}\right)\right.\right. \\
& \left.\left.\left[\left(\frac{8}{3}\right)\left(\ell^{2}-\frac{(q \cdot \ell)^{2}}{q^{2}}\right)-4 \ell^{2}+q^{2}+4 M_{u}\left(\ell_{-}^{2}\right) M_{u}\left(\ell_{+}^{2}\right)\right]\right]-[u \longrightarrow d]\right\},
\end{aligned}
$$

where for the $u$-quark propagator $S_{u}(p)=F_{u}\left(p^{2}\right)\left[\not p+M_{u}\left(p^{2}\right)\right]$ and similarly for the $d$-quark. The quark-vector-meson couplings are estimated from a standard quark model analysis of the nucleon-vector-meson coupling. We find that $g_{q \rho} \simeq\left(3 m_{q} / 5 M_{N}\right) g_{\rho}\left(1+C_{\rho}\right)$ and $g_{q \omega} \simeq\left(m_{q} / M_{N}\right) g_{\rho}\left(1+C_{\omega}\right) \simeq\left(m_{q} / M_{N}\right) g_{\rho}$. With quark masses of $\simeq 400 \mathrm{MeV}$ this gives $g_{q \rho} \simeq 3.9-4.3$ and $g_{q \omega} \simeq 4.8-5.2$ as typical ranges for these couplings. Throughout this work we will use then $g_{q \rho} g_{q \omega}=20.0$.

In the chiral limit, (i.e., with massless quarks and neglecting electromagnetic and weak effects), the pion decay constant can be related to the quark propagator through the Goldberger-Treiman relation (see, e.g., 20] and references therein) to give

$$
f_{\pi}^{2}=(-12 i) \int \frac{d^{4} \ell}{(2 \pi)^{4}} \frac{F\left(\ell^{2}\right) M\left(\ell^{2}\right)}{\ell^{2}-M^{2}\left(\ell^{2}\right)}\left[M\left(\ell^{2}\right)-\frac{1}{2} \ell^{2} \frac{d M}{d \ell^{2}}\left(\ell^{2}\right)\right] .
$$


Factors of three and four follow from colour and spinor traces respectively. (Note that this differs slightly from the expression in Ref. [20 which used a dressed axial-vector vertex instead of the appropriate bare one. The bare vertex avoids double counting problems). Similarly, in the chiral limit (see, e.g., [20] and references therein) we obtain an expression for the quark condensate in terms of the quark propagator

$$
\langle\bar{q} q\rangle \equiv-m_{0}^{3}=-i \int \frac{d^{4} \ell}{(2 \pi)^{4}} \operatorname{Tr} S(\ell)=(-12 i) \int \frac{d^{4} \ell}{(2 \pi)^{4}} F\left(\ell^{2}\right) M\left(\ell^{2}\right),
$$

where the trace is over colour and spinor indices. We use these two results to constrain the quark propagators by fitting them to the experimental values of $f_{\pi}=93 \mathrm{MeV}$ and $\langle q \bar{q}\rangle \equiv m_{0}^{3}=-(225 \mathrm{MeV})^{3}$, (i.e, $\left.m_{0}=225 \mathrm{MeV}\right)$.

Motivated by the confining quark model from the Dyson-Schwinger equation study of Ref. [19] and the need to avoid the artificial quark pair-production threshold encountered by GHT in the timelike region we first consider the following form for the quark propagator [c.f., Eq. (17)]:

$$
F\left(q^{2}\right)=f_{0} e^{-q^{2} / \mu^{2}} \quad \text { and } \quad M\left(q^{2}\right)=m
$$

where $f_{0}$ and $\mu$ are parameters and $m$ is to be chosen as a typical infrared quark mass. This propagator is confining and analytic everywhere in the complex $q^{2}$ plane (i.e., entire). It captures the essential elements of the propagator of Ref. [19] and has the great advantage that with the appropriate choice of gaussian quark-vector-meson form factors we can use Eq. (8) to obtain an analytic expression for the mixing amplitude $\Pi\left(q^{2}\right)$ in both the timelike and spacelike regimes. In practice it is easier to calculate the expressions for $f_{\pi}$ and the condensate $m_{0}$ [i.e., Eqs. (9,10)] after first rotating to Euclidean space. Evaluating these expressions for our analytic (confining) propagator gives

$$
\begin{aligned}
f_{0} & =4 \pi^{2} m_{0}^{3} / 3 m \mu^{4} \\
f_{\pi}^{2} & =\frac{3 f_{0} m^{2} \mu^{2}}{4 \pi^{2}}\left[1-\left(a e^{a} \int_{a}^{\infty} d t \frac{e^{-} t}{t}\right)\right]_{a=(m / \mu)^{2}}
\end{aligned}
$$


Hence we can now fix the parameters $f_{0}$ and $\mu$. Motivated by the infrared values of the dynamically generated quark mass in Dyson-Schwinger equation studies [20] we choose here $m=450 \mathrm{MeV}$ for the "constituent" quark mass. Then substituting the expression for $f_{0}$ into the expression for $f_{\pi}^{2}$ in Eqs. (12) lets us solve for $\mu$ to give $\mu=523.6 \mathrm{MeV}$ and hence we then find $f_{0}=4.432 \times$ $10^{-6} \mathrm{MeV}^{-2}$. Thus all parameters are now constrained.

Since the $u$ - $d$ quark mass difference is commonly estimated to be $\simeq 4 \mathrm{MeV}$ we use $m_{d}-m_{u}=4 \mathrm{MeV}$ throughout this work. Hence the "constituent" masses are $m_{u}=450 \mathrm{MeV}$ and $m_{d}=454 \mathrm{MeV}$ and $f_{0 u}=f_{0}$ and $\mu$ are as determined above. The simplest and most reasonable assumption is that $\mu$ has the same value for the $u$ and $d$ quarks, and hence from Eq. (12) $f_{0 u} / f_{0 d}=m_{d} / m_{u}$. As discussed above in order to facilitate the integration in Eq. (8), we use $F_{q \rho}\left(\ell^{2}\right)=F_{q \omega}\left(\ell^{2}\right)=\exp \left(\ell^{2} / \Lambda^{2}\right)$ with $\Lambda=1 \mathrm{GeV}$, which is a typically reasonable number for the form factor cut-off. We see that these form factors fall off as gaussians in the spacelike region. The integral in Eq. (8) can be performed easily and one obtains for $\Pi\left(q^{2}\right)$

$$
\Pi\left(q^{2}\right)=\frac{3 g_{q \rho} g_{q \omega}}{16 \pi^{2} \lambda^{2}} e^{q^{2} / 2 \mu^{2}}\left\{\left[f_{0 u}^{2}\left[-q^{2}-4 m_{u}^{2}-(4 / \lambda)\right]\right]-[u \rightarrow d]\right\},
$$

where $\lambda \equiv 2\left[\left(1 / \Lambda^{2}\right)+\left(1 / \mu^{2}\right)\right]$. The mixing amplitude has thus been calculated for all timelike and spacelike $q^{2}$ for the analytic (confining) case.

We can also numerically evaluate $\Pi\left(q^{2}\right)$ for numerical solutions of quark Dyson-Schwinger equations. These solutions are fitted to both $f_{\pi}$ and the quark condensate in the usual way 20] and in addition have the correct perturbative asymptotic behaviour in the spacelike (Euclidean) region. Unfortunately it is not yet known how to continue these solutions into the timelike regime in the general case. We have introduced a $4 \mathrm{MeV}$ mass splitting of the infrared mass and have solved for the $u$ and $d$ propagators in the usual way [20]. The quark-vector-meson couplings $\left(g_{q \rho}\right.$ and $\left.g_{q \omega}\right)$ have already been deduced and for the corresponding form-factors we choose the form used by 
GHT for ease of comparison, i.e., $F_{q \rho}\left(\ell^{2}\right)=F_{q \omega}\left(\ell^{2}\right)=1 /\left[1-\left(\ell^{2} / \Lambda^{2}\right)\right]$. We again use $\Lambda=1 G e V$. We can now use Eq. (8) to evaluate $\Pi\left(q^{2}\right)$ for a typical Dyson-Schwinger equation solution in the spacelike region.

Finally, for comparison we repeat the calculation with the numerical DysonSchwinger solution replaced by a free quark propagator with artificially heavy quarks $\left(m_{u}=600 \mathrm{MeV}\right.$ and $\left.m_{d}=604 \mathrm{MeV}\right)$. This calculation is then identical to that of GHT except that now there is no troublesome quark threshold between the spacelike region and the $\omega$-mass point where the mixing amplitude is actually measured.

In order to understand the importance of the momentum dependence of the mixing amplitude on the nucleon-nucleon potential due to vector meson exchange, we consider the static, central part of this potential. Besides a central potential, the exchange of a vector boson gives rise to spin-orbit, spinspin and tensor potentials. Although the spin-dependent potentials are the relevant ones for the TRIUMF and IUCF experiments, the radial part of these potentials will be directly related to that of the central one. The coordinate space $N N$ potential due to $\rho \omega$-mixing, $V(r)$, is the Fourier transform of $V(q)$ in Eq. (5), where $q^{0}=0$ and where we here define $q \equiv|\vec{q}|$

$$
\begin{aligned}
V(r) & =\frac{1}{2 \pi^{2} r} \int_{0}^{\infty} d q q \sin (q r) V(q) \\
& =\frac{1}{2 \pi^{2} r} \int_{0}^{\infty} d q q \sin (q r) \frac{-g_{\rho} g_{\omega} F_{N \rho}\left(-q^{2}\right) F_{N \omega}\left(-q^{2}\right) \Pi\left(-q^{2}\right)}{\left(q^{2}+m_{\rho}^{2}\right)\left(q^{2}+m_{\omega}^{2}\right)}
\end{aligned}
$$

In Fig. 1 we show the momentum-dependence of the $\rho^{0}-\omega$ mixing amplitude for the three quark calculations described here, i.e., the confining (analytic), Dyson-Schwinger equation (dse), and heavy-free (free) cases. Also shown for comparison are recent hadronic calculations 15, where an essentially parameter free calculation was made using an $N \bar{N}$ loop and the $n-p$ mass difference. The two hadron-model curves differ in that one (hadron_ff) has the usual Bonn form factor applied at the vertices of the nucleon loop in 
the spaceklike region as well as at the meson-nucleon vertices. We see that all calculations appear capable of fitting the data with typical parameters and that the momentum dependence is remarkably similar in each case. We see strong momentum-dependence of the mixing amplitude and a change in sign in the vicinity of $q^{2}=0$. This is also what was seen in the recent QCD sum rules study [16], although there $\Pi\left(q^{2}\right)$ was seen to change sign in the timelike region at $q^{2} \simeq 0.25-0.40 \mathrm{GeV}^{2}$ rather than the spacelike region.

In Fig. 2 we show the momentum-space potential $V(q)$ given in Eq. (5) for the various models. Also for comparison we show the result obtained when the usual assumption of momentum-independence of the mixing amplitude is made. In Fig. 3a) we show the corresponding coordinate-space potentials $V(r)$ given by Eq. (14) for each of these cases. Fig. 3b) has an enhanced vertical scale in order to show the location of nodes in the potential $V(r)$. We see that all calculations predict strong suppression of the $\rho^{0}-\omega$ mixing potential compared with that resulting from the usual momentum-independent assumption for the mixing amplitude. We see that the Dyson-Schwinger propagator and the hadronic models predict the opposite sign at small $r$ and have nodes between 0.5 and $0.8 \mathrm{fm}$, whereas the heavy free quark and confining (analytic) quark cases have the same sign as the usual assumed potential, but are very strongly suppressed. It is interesting to note that the heavy free quark case presented here is identical to the case studied by GHT with the exception of having heavier (i.e., $600 \mathrm{MeV}$ ) quarks. We see that this increase in mass has removed the node in $V(r)$ for the free propagator case. Given the variety of approaches to the calculations, the results are remarkably similar.

The possible implications for our understanding of CSB are far-reaching. For example, in the case of the $\rho-\omega$ mixing contribution to the class IV CSB in $n$ - $p$ elastic scattering[6]-[10] there is a competition between large- $r$ fall-off in the potential and short-distance suppression of the distorted $N-N$ wavefunc- 
tion. The result is that the conventional contribution peaks around $0.9 \mathrm{fm}$. It is interesting that this is the region where nodes occur. All models predict at least strong suppression in this region. It is clear that the previously assumed theoretical understanding of charge-symmetry breaking phenomena is now questionable. The models and treatments to date appear to be in overall agreement. The crucial question is whether these conclusions will survive future, more elaborate examinations.

\section{Acknowledgements}

We are thankful to K. Yazaki for for a helpful discussion. This work was supported by the Australian Research Council (AWT and AGW) and CNPq (Brasil) (GK). AGW and GK were also partially supported by the U.S. Department of Energy through Contract No. DE-FG05-86ER40273 and by the Florida State University Supercomputer Computations Research Institute which is partially funded by the Department of Energy through Contract No. DE-FC05-85ER250000. 


\section{References}

[1] G.A. Miller, B.M.K. Nefkens and I. Slaus, Phys. Rep. 194 (1990) 1.

[2] E.M. Henley and G.A. Miller, in "Mesons and Nuclei," eds. M. Rho and D.H. Wilkinson (North Holland, 1979); A. Gersten et al., Few-Body Systems 3 (1988) 171.

[3] S.A. Coon and R.C. Barrett, Phys. Rev. C 36 (1987) 2189.

[4] J.A. Nolen, Jr. and J.P. Schiffer, Ann. Rev. Nucl. Sci. 19 (1969) 471.

[5] P.G. Blunden and M.J. Iqbal, Phys. Lett. B198 (1987) 14.

[6] R. Abegg et al., Phys. Rev. Lett. 56 (1986) 2571; Phys. Rev. D 39 (1989) 2464.

[7] L.D. Knutson et al., Nucl. Phys. A508 (1990) 185c; L.D. Knutson et al., Phys. Rev. Lett. 66 (1991) 1410; S.E. Vigdor et al. Phys. Rev. C 46 (1992) 410.

[8] B. Holzenkamp, K. Holinde and A. W. Thomas, Phys. Lett. B195 (1987) 121.

[9] G.A. Miller, A.W. Thomas and A.G. Williams, Phys. Rev. Lett. 56 (1986) 2567; A.G. Williams, A.W. Thomas, and G.A. Miller, Phys. Rev. C 36 (1987) 1956.

[10] M. Beyer and A.G. Williams, Phys. Rev. C 36 (1987) 1956.

[11] T. Goldman, J.A. Hendersen and A.W. Thomas, Few Body Systems 12 (1992) 123.

[12] K. Maltman and T. Goldman, Modelling the Off-Shell Dependence of $\pi^{0}-\eta$ Mixing with Quark Loops, Los Alamos preprint LA-UR-92-2910. 
[13] K. Maltman, $q^{2}$-Dependence of Meson Mixing in Few-Body Charge Symmetry Breaking: $\pi^{0}-\eta$ mixing to One Loop in Chiral Perturbation Theory, Los Alamos preprint LA-UR-92-2549.

[14] J. Piekarewicz, Off-shell Behavior of the $\pi-\eta$ Mixing Amplitude, Florida State University preprint FSU-SCRI-030893.

[15] J. Piekarewicz and A.G. Williams, The Momentum Dependence of the $\rho-\omega$ Mixing Amplitude in a Hadronic Model, Florida State University preprint FSU-SCRI-122192, to appear in Phys. Rev. C.

[16] T. Hatsuda, E.M. Henley, Th. Meissner, and G. Krein, The Off-Shell $\rho-\omega$ Mixing in the QCD Sum Rules, Institute for Nuclear Theory (Seattle) preprint INT93-00-36.

[17] R. Machleidt, K. Holinde and Ch. Elster, Phys. Rep. 149 (1987) 1.

[18] C.D. Roberts, A.G. Williams, and G. Krein, Int. J. Mod. Phys. A7 (1992) 5607.

[19] C.J. Burden, C.D. Roberts and A.G. Williams, Phys. Lett. 285 (1992) 347.

[20] A.G. Williams, G. Krein and C.D. Roberts, Ann. Phys. (NY) 210 (1991) 464; F.T. Hawes and A.G. Williams, Phys. Lett. B268 (1991) 271. 
Fig. 1 The $\rho-\omega$ mixing amplitudes calculated in a variety of models, (see text). Also shown for comparison is the experimentally extracted amplitude at the $\omega$-mass-shell point and the usual assumed $q^{2}$-independent behaviour.

Fig. 2 The $\rho-\omega$ momentum space potentials, $V(q)$, for the mixing amplitudes shown in Fig. 1. $V(q)$ is defined in the text.

Fig. 3 The $\rho-\omega$ coordinate space potentials, $V(r)$, for the mixing amplitudes shown in Fig. 1. $V(r)$ is defined in the text. We show the full range of the potentials in a), while in b) an enhanced vertical scale is used to show in detail how the potential changes sign in some cases. 


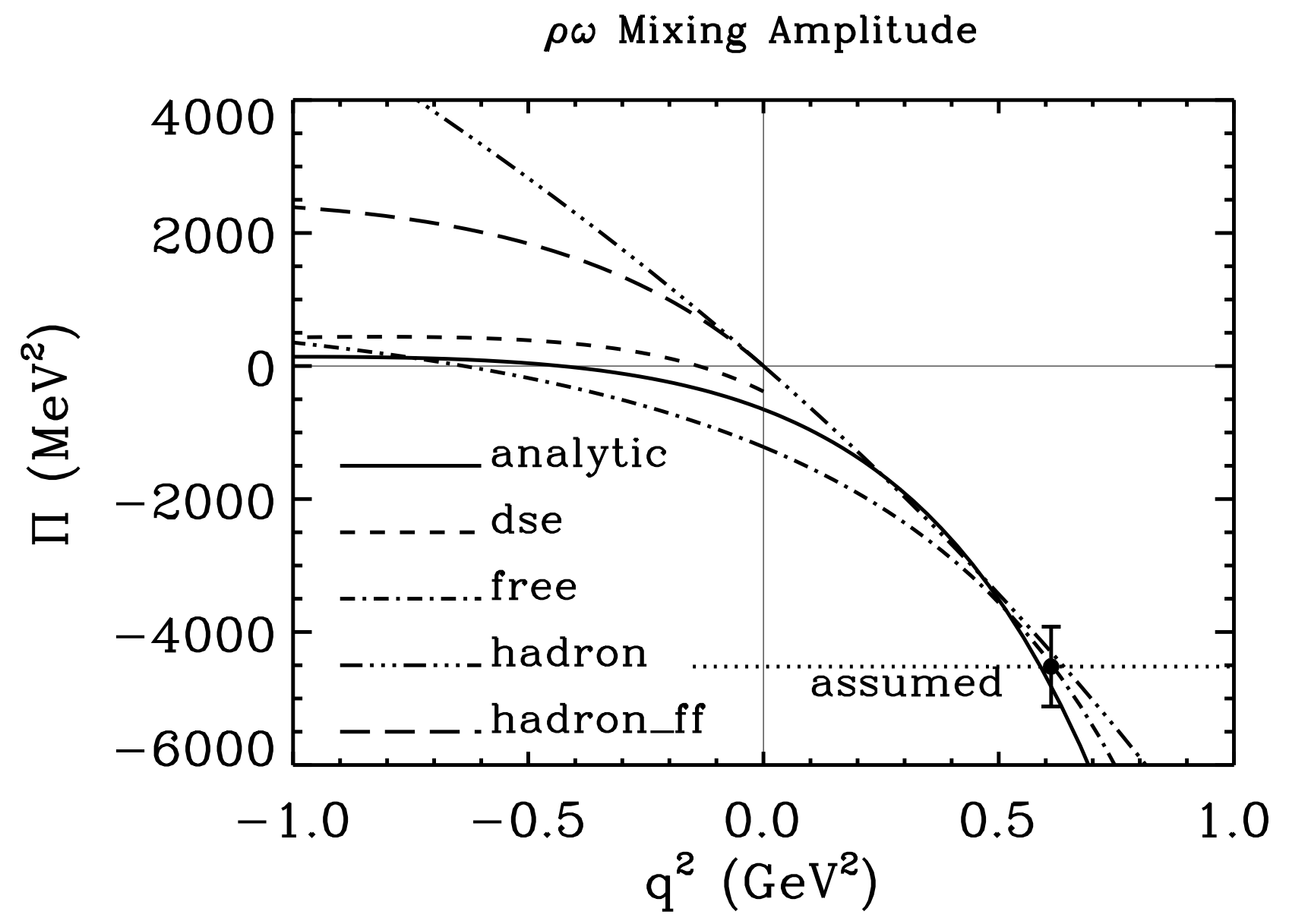

Figure 1 


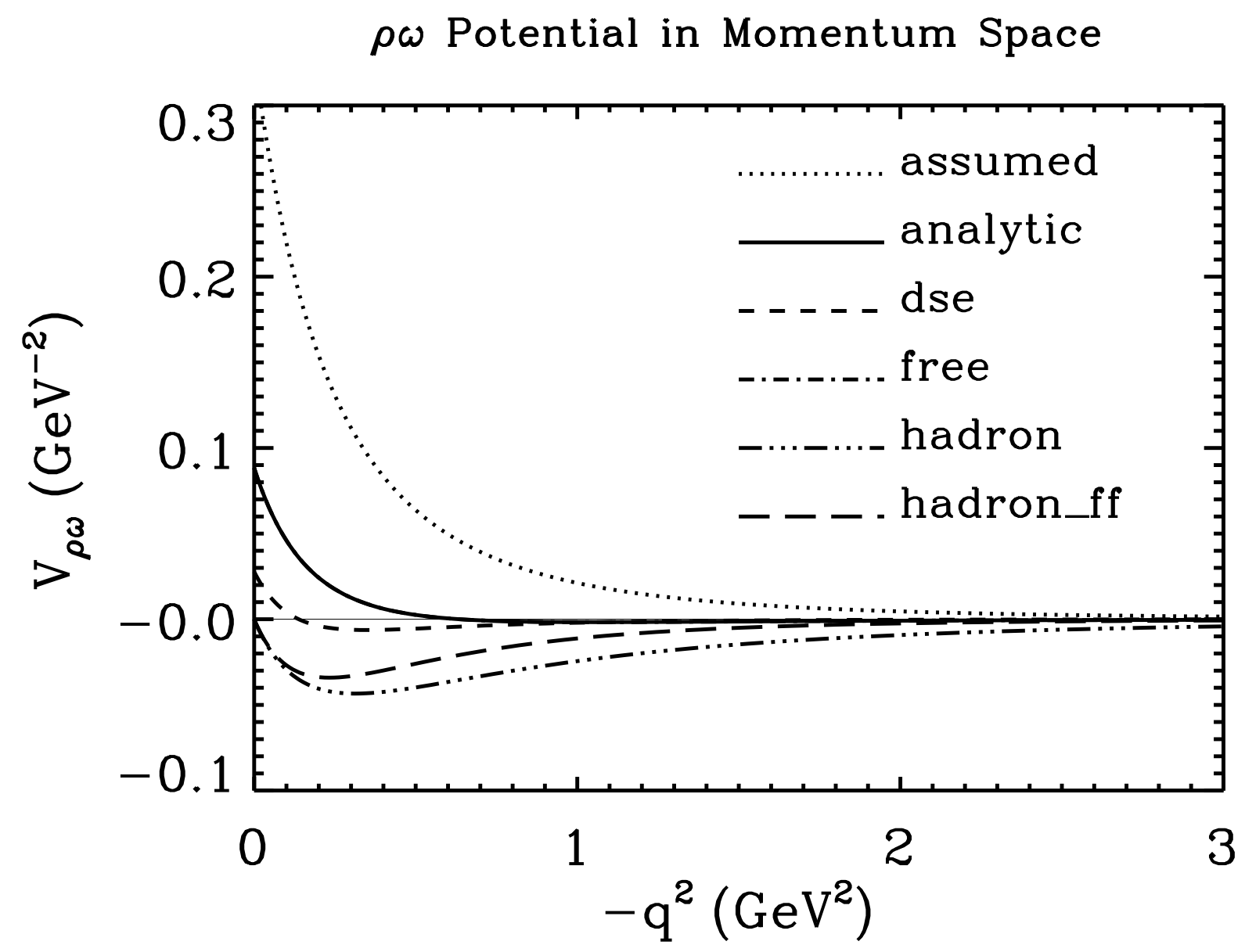

Figure 2 


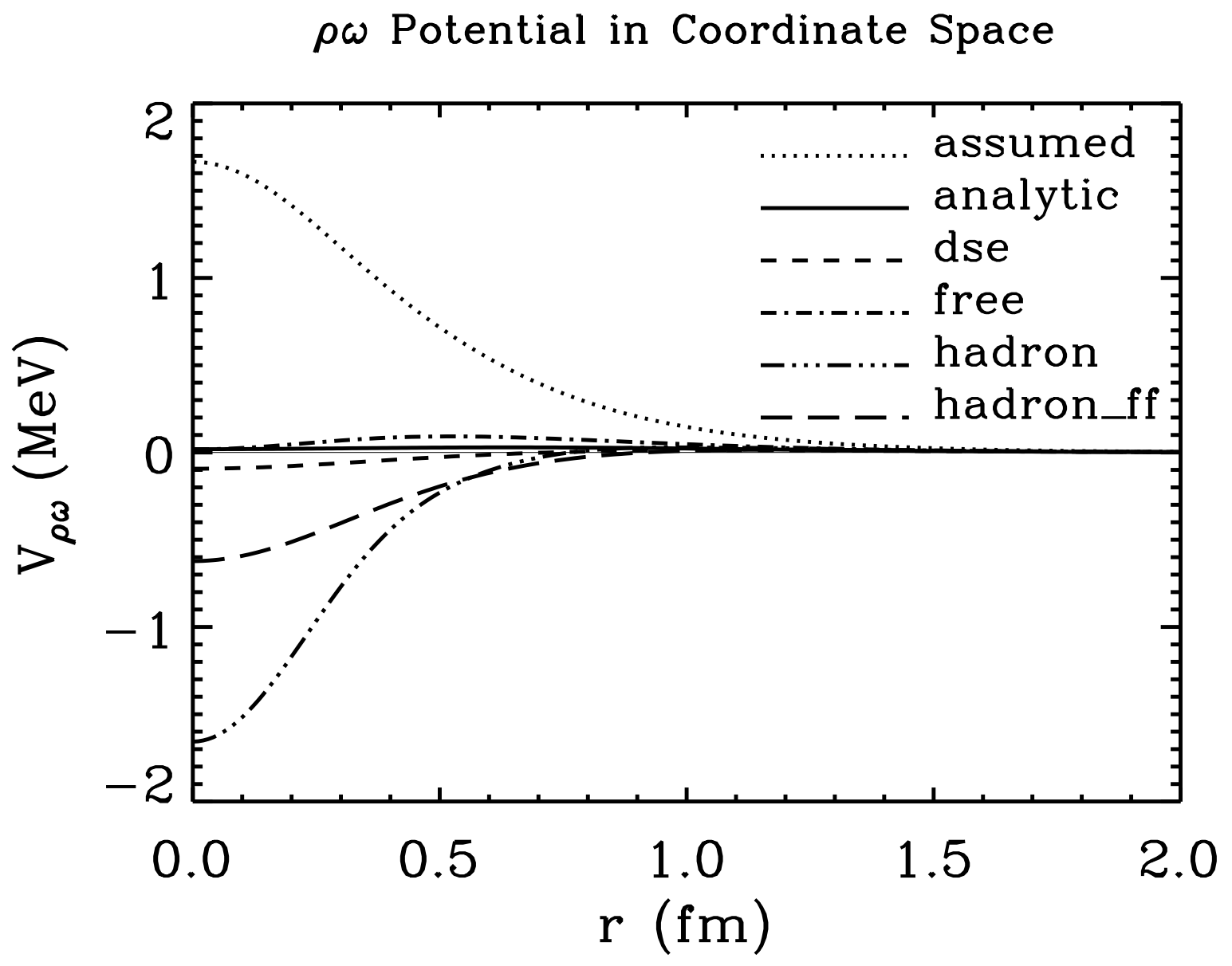

Figure 3a) 


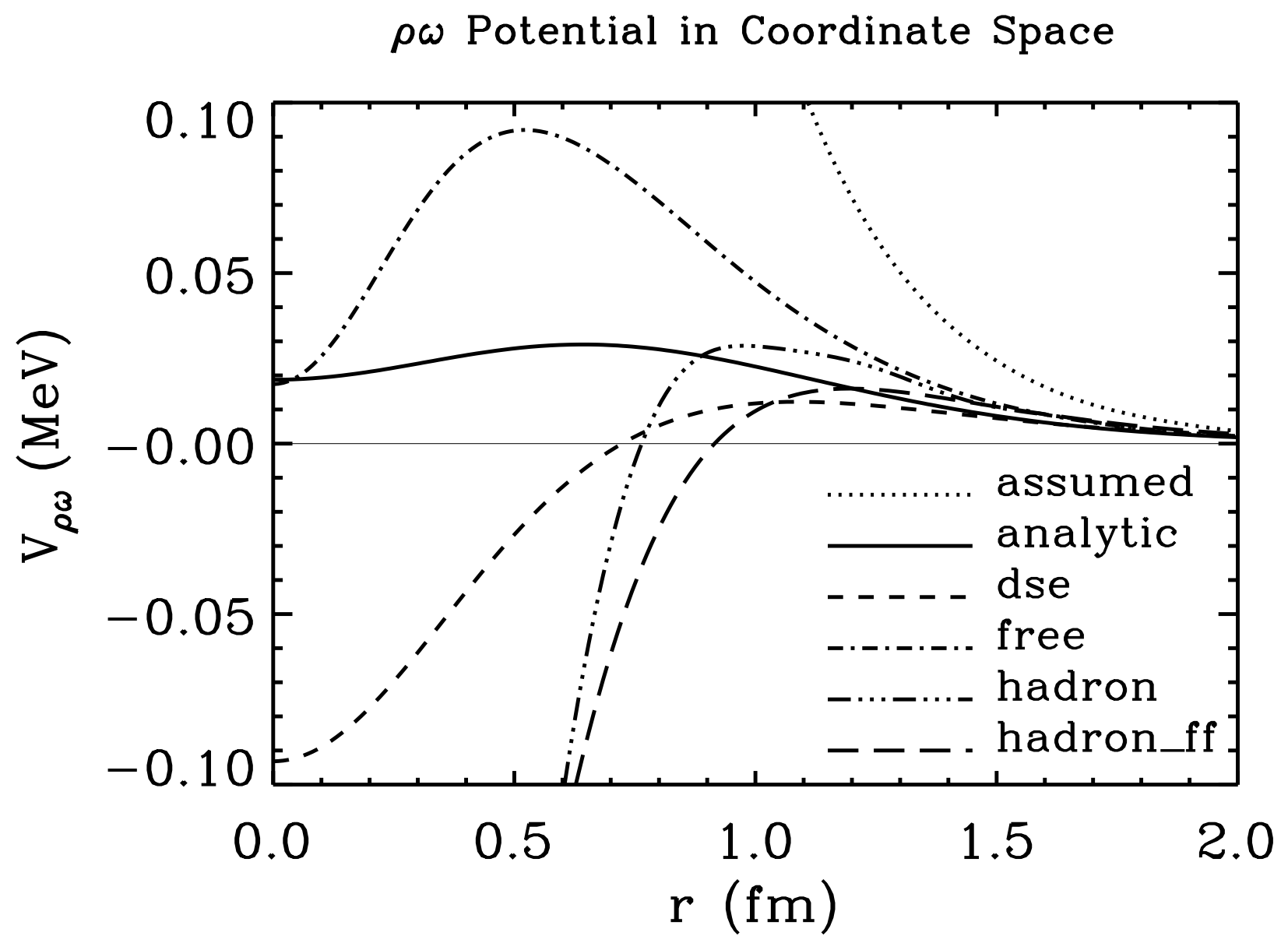

Figure 3b) 